\title{
Shock structures in stellar winds resulting from orbital motions of the star
}

\author{
Jorge Cantó, Alejandro Raga, Gloria Koenigsberger, \\ and Edmundo Moreno \\ Instituto de Astronomía, UNAM, Ap. 70-264, 04510 D.F., México
}

\begin{abstract}
We study the problem of a wind ejected from a star which orbits around a (wind-less) companion star. The orbital motion induces the formation of a spiral shock structure in the wind. An analytic formulation of the problem allows us to obtain the distance from the star at which the shock waves appear, and also the locus of the spiral, two-shock structure (i.e., the working surface). The full, three-dimensional structure of the working surface is found to have a spiral intersection with the orbital plane, and a cylindrical "hole" around the polar axis.
\end{abstract}

\section{Shocks in a wind from an orbiting star}

Let us consider a star with an isotropic stellar wind with terminal velocity $v_{0}$ which has a windless binary companion. The binary system has circular orbits, with a period $P$ (or angular velocity $\omega=2 \pi / P$ ), and an orbital velocity $v_{c}$.

Along a given direction in the plane of the orbit, and far away from the binary system, the velocity of the wind is given by:

$v=v_{0}-v_{c} \sin (\omega \tau)$

where $\tau$ is the time elapsed since the star crossed the given direction. Using this velocity variation, one can directly apply the results of Cantó, Raga \& D'Alessio (1999).

\section{Flow in the plane of the orbit}

For each ejection time $\tau$, we have an ejection direction $\psi=\omega \tau$. The position of the working surface at time $t$ is given by

$r_{w s} \approx v_{0}\left(t-\tau-\frac{\pi}{\omega}\right), \quad$ with $\tau \leq t-t_{c}$

In a frame of reference rotating with angular velocity $\omega$, the locus of the working surfaces is given by

$r_{w s} \approx-\frac{v_{0}}{\omega}\left(\psi^{\prime}+\pi\right), \quad$ where $\psi^{\prime} \equiv \omega(\tau-t) \leq-\omega t_{c}$

The above equation shows that the locus of the working surface on the orbital plane is an Arquimedes' spiral. This spiral working surface first appears at a radius $r_{c} \approx v_{0}^{2} / \omega v_{c}$, and the separation between the spiral arms is $\Delta r_{w s} \approx$ $2 \pi v_{0} / \omega$. Note that in the observer's frame of reference, this spiral pattern rotates with the angular velocity $\omega$.

\section{Three-dimensional flow}

Consider now a direction with polar angle $\theta$. For this direction, the rotational velocity is $v_{c} \sin \theta$. The position of the working surface is the same as above, but with 


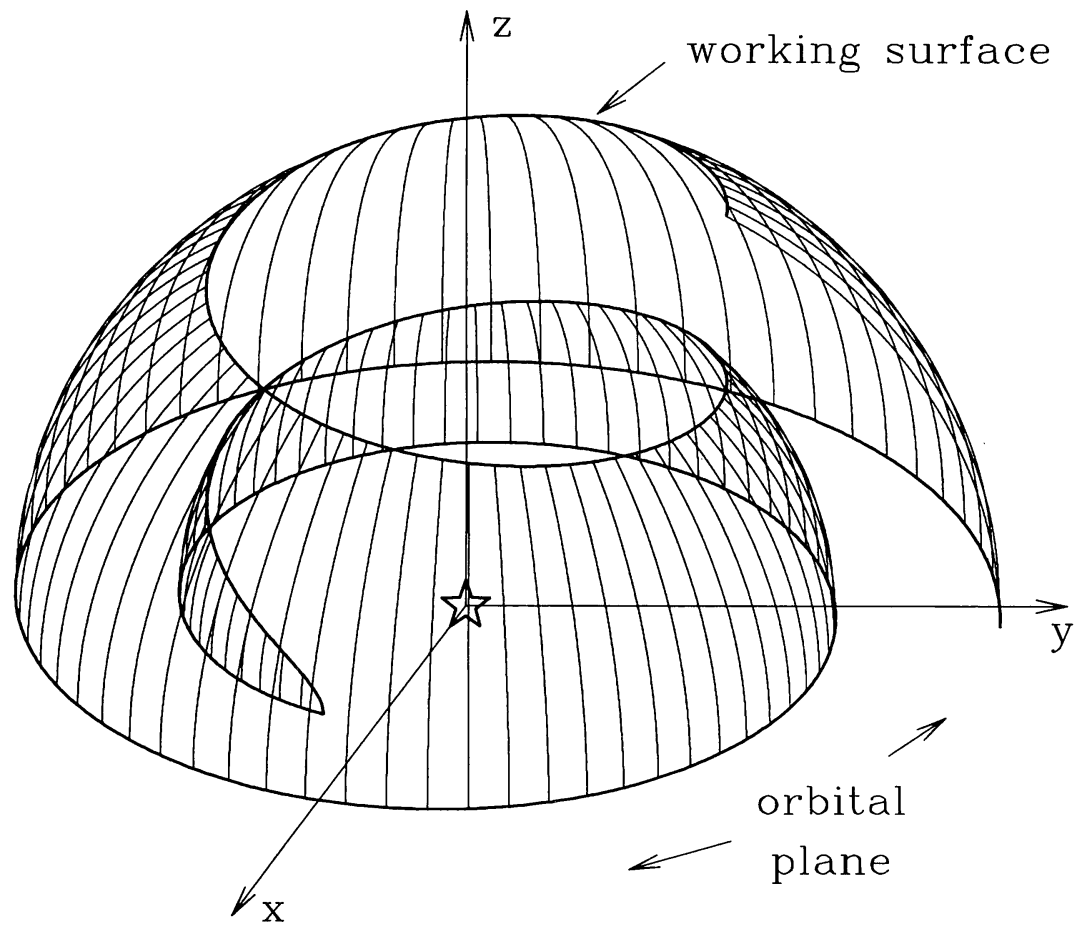

Figure 1. Representation of the two-shock surface generated in a stellar wind as a result of an orbital motion of the star

$t_{c}=\frac{1}{\omega}\left(\pi+\frac{v_{0}}{v_{c} \sin \theta}\right)$

The working surface is then given in the rotating (cartesian) frame of reference by the parametric standard equations. An example of the structures that can be produced is shown in Figure 1.

\section{Discussion}

We have presented a simple, analytic solution for the formation of discontinuities in a stellar wind as a result of an orbital motion of the wind source around a windless companion. This solution in principle can be used to model the structure of bubbles and/or ionized nebulae around WR stars or young, low mass stars.

Acknowledgments. We acknowledge support from the CONACyT grant 26833-E.

\section{References}

Cantó, J., Raga, A.C., D’Alessio, P. 1999, MNRAS submitted. 